Original

\title{
Las pruebas epicutáneas de contacto en medicina laboral
}

\section{Patch testing in occupational medicine}

\section{Carolina Granados Rincón', Nelly Machado Benavides², Ana Rita Rodrigues-Barata ${ }^{3}$, Luis Conde-Salazar Gómez ${ }^{3}$}

1. Hospital "Lluís Alcanyís" de Xàtiva. Escuela Valenciana de Estudios de la Salud. Valencia. España.

2. Servicio de Dermatología, Universidad San Francisco. Hospital Carlos Andrade Marín (USFQ-HCAM). Quito. Ecuador.

3. Servicio de Dermatología Laboral. Escuela Nacional de Medicina del Trabajo. Instituto de Salud Carlos III. Madrid. España.

Recibido: 17-12-12

Aceptado: 10-01-13

\section{Correspondencia}

Carolina Granados Rincón

Calle Trobat 18 Piso 2

46800 Xàtiva (Valencia). España.

Teléfono: +34699618818

Correo electrónico: carolgigi26@gmail.com

Resumen

Dentro de la Medicina Laboral las dermatosis ocupacionales suponen entre el 30-60\% del total de enfermedades profesionales y, dentro de ellas el $80-90 \%$ son dermatitis de contacto. Este alto número no se ve reflejado en datos oficiales debido a que en muchas ocasiones, al ser condiciones de rápida resolución o de escasa repercusión sistémica, no son declaradas. En relación a las dermatitis de contacto, la forma más frecuente en el entorno laboral es la forma alérgica y la que preferentemente se declara, al ser un proceso que en muchas ocasiones constituye motivo de incapacidad. Para el abordaje diagnóstico y correspondiente asociación con el entorno de trabajo es imprescindible realizar una historia clínica laboral completa junto con la descripción de las lesiones, y la utilización de métodos diagnósticos distintos a los de otros procesos no cutáneos, como son las pruebas de parche (patch test) o pruebas epicutáneas. Constituyen un método simple, accesible y seguro para llegar al diagnóstico de las dermatitis alérgicas de contacto. Incluyen una batería estándar, propia de un país donde se trabaja, ampliada de baterías específicas en relación con el perfil, las tareas y los productos manipulados por el trabajador. La prevención en el trabajo es el único tratamiento etiológico para la sustitución y control de los riesgos laborales relacionados con las dermatosis profesionales.

Med Segur Trab (Internet) 2013; 59 (230) 74-84

Palabras clave: Dermatosis ocupacional, pruebas epicutáneas, pruebas de parche, dermatitis de contacto, dermatología ocupacional. 


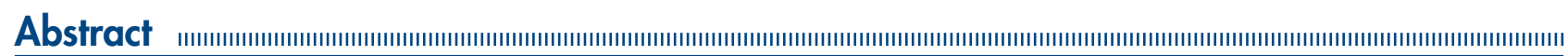

In occupational medicine, occupational dermatoses account for 30 to $60 \%$ of the total amount of occupational diseases and, within 80 to $90 \%$ of them correspond to contact dermatitis. This high number is not reflected in official data because in most cases, as conditions of rapid resolution or low systemic impact, they are not declared. Regarding contact dermatitis, the most common type in the workplace is the allergic type, which preferably is declared, because often results in disability. For the correct diagnosis and association with the work environment is essential to make a full history of the patient' occupational background, correct description of the skin lesions, and the use of diagnostic methods other than those of non-cutaneous processes, such as patch testing. They provide a simple, accessible and safe method for the diagnosis of allergic contact dermatitis. They include a standard national baseline series, proper of the country where the patient works, and additional extended series, specific to the occupation performed by the worker. Prevention at work is the only etiological treatment for replacement and control of occupational hazards associated with occupational skin diseases.

Med Segur Trab (Internet) 2013; 59 (230) 74-84

Key words: Occupational skin disease, patch testing, skin test, contact dermatitis, occupational dermatology. 


\section{INTRODUCCIÓN}

Las dermatitis de contacto (DC) constituyen alrededor del 90\% del amplio espectro de las dermatosis profesionales, y se pueden dividir en dos formas: la dermatitis de contacto irritativa, que comprende el $80 \%$ de los casos, y la dermatitis de contacto alérgica que es la responsable del $20 \%$ de los casos ${ }^{1}$. El resto de dermatosis ocupacionales se pueden enumerar como: las urticarias de contacto, las foliculitis, el cloroacné, las leucodermias, las esclerodermias, las infecciones bacterianas y micóticas así como las neoplasias cutáneas ${ }^{2}$. No existen estimaciones precisas en relación a la prevalencia e incidencia de las dermatosis profesionales por la frecuente equivocación a la hora de establecer el diagnóstico de accidente y enfermedad ocupacional, y de distinguir entre las dermatitis de contacto con otras patologías cutáneas ${ }^{3}$. Las DC profesionales están consideradas como la enfermedad profesional de mayor incidencia, con cifras superiores al 30\%, siendo las patologías más notificadas y las que requieren más compensación económica ${ }^{3,4}$. Informes recientes de proyectos de vigilancia de enfermedades cutáneas, Epiderm y Opra, sugieren una tasa de incidencia de 13 por 100000 por año, y una prevalencia de 15 por 10000 trabajadores incluidos en estos estudios ${ }^{5}$. La localización más frecuente de las dermatosis ocupacionales son las manos, con un $80-90 \%$ de los casos $^{3}$. Existen diversas profesiones que difieren en el riesgo de desarrollar enfermedades cutáneas de origen ocupacional. Entre las de alto riesgo están los peluqueros (tasa anual de 120/100000), trabajadores de artes gráficas (tasa anual de 71/100000), operarios de maquinaria y herramientas (tasa anual 56/100000), trabajadores de plantas químicas y petroleras (tasa anual de 45/100000), ensambladores (tasa anual de 35/100000) y montadores de herramientas para maquinaria (tasa anual de 34/100000) 5 .

\section{Conceptos básicos}

En 1939 la Asociación Médica Americana define la dermatosis profesional como "una afectación de la piel en la que puede encontrarse que el trabajo es su causa fundamental o un factor que contribuye a ella". Esta definición fue modificada en el X Congreso Latinoamericano de Dermatología de 1983 como "toda alteración de la piel, mucosas y anexos, directa o indirectamente causada, condicionada, mantenida o agravada por todo aquello que sea utilizado en la actividad profesional o exista en el ambiente de

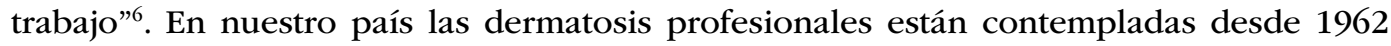
(R.D. 792/1961 del 13 de Abril), como "toda enfermedad de la piel de origen físico o químico, bien sea por irritantes primarios o por sensibilizaciones cutáneas que obliguen a una interrupción del trabajo permanente o recidivante" ${ }^{3,6}$.

Por tanto, dermatosis profesional desde el marco jurídico se definiría como "aquellas que se encuentran recogidas en la legislación del país donde se trabaja". Por esta razón hoy día, para clasificar una dermatosis como enfermedad ocupacional se tiene que cumplir una serie de hechos ${ }^{3,7}$ :

1. Historia clínica concordante.

2. La localización de las lesiones deben corresponder en zonas concordantes con la forma y tipo de trabajo.

3. Pruebas de contacto positivas o pruebas de aplicación abierta positivas con relevancia presente.

4. Estudios en controles voluntarios con concentraciones no irritantes cuando se desconozcan las concentraciones adecuadas.

5. Mejoría cuando no se trabaja o cuando se evite el contacto con el alérgeno implicado.

Por este motivo, es especialmente transcendental el estudio y la implicación de los Médicos del Trabajo y los Dermatólogos en la declaración de las dermatosis profesionales ya que ésta labor debe ser efectuada con rigor y precisión, profundizando en un 
conocimiento exacto y detallado de la actividad laboral y del perfil del trabajador, porque puede acarrear consecuencias importantes sobre la vida profesional del paciente y por la enorme compensación económica que puede suponer su diagnóstico ${ }^{7,8 .}$

En relación a la DC, se puede definir como una reacción inflamatoria aguda o crónica de la piel al contacto directo con agentes nocivos, en concentración y período de tiempo suficiente para provocar una alteración con morfología claramente objetivable ${ }^{9,10}$. En bastantes ocupaciones la piel está expuesta a diversos agentes químicos, biológicos, físicos y mecánicos, y el daño que pueden originar dependerá de la capacidad para de recuperación cutánea, que es variable de un individuo a otro ${ }^{11}$.

\section{Aspectos históricos}

La historia del conocimiento de la DC y su evolución a lo que se conoce hoy en día está íntimamente vinculada con el advenimiento de las pruebas epicutáneas o patch test. Las DC se conocen desde hace siglos, cuando ya se empezaba a describir lesiones ocasionadas por sustancias en las que se guardaba una relación con el trabajo. Por ejemplo, en tiempos del Emperador Augusto se hacía mención a las úlceras producidas por cáusticos. Posteriormente Paracelso describió los efectos perjudiciales que ejercían algunas profesiones en la piel; Ellembog y Agrícola estudiaron las acciones sobre la piel y tegumentos de humos de metales como el cadmio, el arsénico o el cobalto, constituyendo un gran aporte al conocimiento de las dermatitis ocasionadas por estos elementos ${ }^{7}$. En 1715, Bernardo Ramazzini, describe las enfermedades profesionales que existían en aquel tiempo y plantea diversas medidas para su prevención y tratamiento. En 1798, Willan también publicó en su libro "Descripción y Tratamiento de las Enfermedades Cutáneas" una asociación entre cuadros cutáneos con profesiones diversas ${ }^{7}$.

En el siglo XIX Josef Jadasssohn realiza grandes aportaciones con su la introducción de las pruebas epicutáneas, cuya técnica fue mejorada por Bruno Bloch, que se logró expandir los hallazgos de Jadassohn ${ }^{12}$. Con la introducción de las técnicas de las pruebas de parche se lograron grandes avances en el estudio y tipificación de las dermatosis de origen ocupacional, lo cual significó un paso importante en la investigación de la prevención y tratamiento de las mismas. Paralelamente, con el auge de la revolución industrial, se empieza a prestar importancia a las alteraciones de la piel en el ámbito ocupacional relacionadas con las sustancias que eran capaces de ocasionarlas, siendo mencionadas en el libro publicado por Prosser White en 1934 "The dermatoses or Occupational Affections of the Skin"12. Posteriormente aparecen múltiples escritos $y$ tratados de diversos autores como Sulzberg, Bonnevie, Schwartz, Tulipan, Adams, Foussereau y Peck sobre esta rama de la dermatología. A partir de la segunda guerra mundial se crearon clínicas de contacto, la primera se llamó Saint John Hospital de Londres-1953 ${ }^{13}$. En 1962 se forma el primer Commitee for Standarization of Routine Patch Testing, que aboca a la creación en 1967 del International Contact Dermatitis Research Group (ICDRG) ${ }^{9,13}$, con lo cual se obtiene una visión clara y concreta acerca del manejo de las pruebas epicutáneas. En nuestro país en 1976 se creó el Grupo Español de Investigación en Dermatitis de Contacto (GEIDAC), que ha desarrollado una magnifica labor en ésta área. En 1992 la batería estándar contenía a 24 haptenos; en el 2008, tras una revisión realizada por las organizaciones mundiales de dermatitis de contacto alérgicas, se incluyeron a 29 sustancias. En España se emplea por consenso la batería Estándar de la GEIDAC (Tabla I). 
Tabla I. Batería Estándar del GEIDC 2008

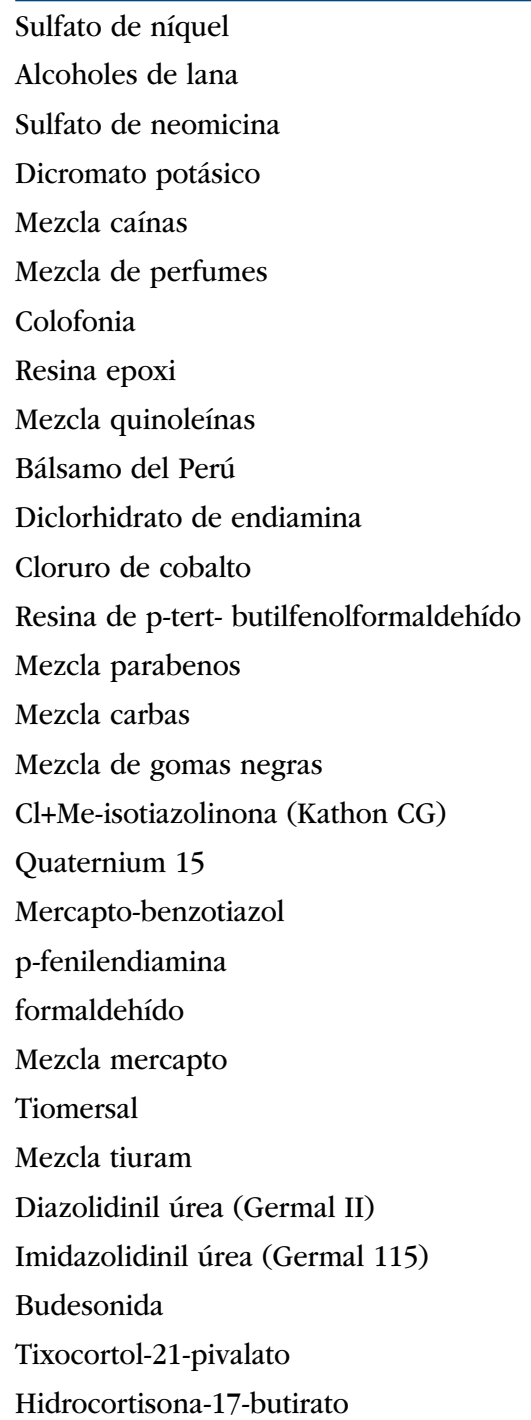

\section{Bases Teóricas e Inmunológicas}

El conocimiento de la fisiopatología de la DC y los mecanismos involucrados permiten distinguir los dos tipos: la alérgica y la irritativa ${ }^{14}$. La DC irritativa es una reacción inflamatoria causada por la acción citotóxica directa del agente agresor a las células de la epidermis y la dermis, sin la producción de anticuerpos específicos, en contraste con la DC alérgica que es una reacción de hipersensibilidad retardada, mediada por células $\mathrm{T}$ y que requiere una sensibilización previa ${ }^{1,15}$.

Las pruebas epicutáneas o pruebas de parche fueron desarrolladas para confirmar o ratificar la causa de una DC alérgica. Constituyen un método sencillo para reproducir la reacción de hipersensibilidad retardada o tipo IV de la piel frente a un alérgeno ${ }^{12}$. En esta reacción los linfocitos $\mathrm{T}$ sensibilizados tienen un contacto secundario con el antígeno, el cual esta usualmente en forma de hapteno conjugado con una proteína y presentado por la célula presentadora de antígeno de la epidermis, la célula de Langerhans ${ }^{5}$. Por lo tanto, las pruebas de parche han sido consideradas el mejor método para distinguir la DC alérgica de la DC irritativa ${ }^{16}$. Sin embargo, es de conocimiento común que la constatación de las reacciones positivas a un determinado alérgeno, obliga a una búsqueda minuciosa de su relevancia. Por esta razón, es muy importante realizar una historia clínico-laboral exhaustiva, explorar posibles agentes de contacto, determinar la localización y evolución 
de la dermatosis, e individualizar la prueba de parche según la historia y perfil del trabajador ${ }^{17,18}$.

\section{Presentación Clínica}

Tanto las DC irritativas como las DC alérgicas suelen comenzar en su fase aguda con eritema pruriginoso y edematoso, acompañándose en ocasiones de vesículas. El eritema y el edema persisten en las fases subagudas, aunque, la vesiculación empieza a hacerse menos visible, siendo reemplazada por escoriación, liquenificación, supuración, fisuración y descamación ${ }^{1,3}$. La DC irritativa tiende a permanecer localizada en el área de contacto mientras que la DC alérgica tiene propensión a diseminarse a sitios distantes, tanto por el proceso de autoeczematización o a través del fenómeno de DC sistémica ${ }^{1}$. Las manos están afectadas en un $80-90 \%$ de los casos ocupacionales. Los brazos pueden estar afectados si no están debidamente protegidos, mientras que la cara y el cuello están afectados si hay exposición a polvos o vapores (mecanismo aerotransportado o airborne) ${ }^{5}$.

Mathias propuso una serie de criterios objetivos para la correcta identificación de la DC ocupacional. Si cuatro de estos criterios están presentes, el médico puede concluir que la DC es de probable origen ocupacional ${ }^{1,19}$ (Tabla II).

Tabla II. Criterios MATHIAS para las dermatitis de contacto de origen laboral

1. Cuadro clínico consistente con dermatitis de contacto.

2. Exposición laboral a potenciales alérgenos o irritantes cutáneos.

3. Distribución Anatómica coherente con la forma de la exposición cutánea en el trabajo.

4. Relación temporal entre la exposición y el inicio de la dermatitis de contacto.

5. Exclusión de exposiciones no laborales como posibles causas.

6. Mejoría del eczema al cesar la exposición.

7. Las pruebas epicutáneas o prick test implican una exposición laboral específica.

Las DC aparecen a cualquier edad pero tienen un pico en los extremos de la vida laboral, ya sea al inicio o final de la misma. En panaderos y peluqueros aparecen de forma temprana, en los primeros años del inicio de la profesión. En trabajadores de la construcción que manipulan cemento, las dermatitis por cromo requieren algunos años para desarrollarse. La DC irritativa acumulativa aparece después de muchos años de exposición 5 .

\section{MATERIAL Y MÉTODOS}

\section{Metodología y problemas prácticos}

Las pruebas de contacto o patch test están consideradas como un método sencillo y de fácil aplicación, con la ventaja sobre las técnicas in vitro de ser llevado a cabo en la propia piel $^{20}$. Tienen una sensibilidad y especificidad estimada del $70 \%$, que puede variar según el hapteno estudiado ${ }^{18}$. Como otros test clínicos, el resultado de las pruebas de contacto puede estar afectado por un gran número de factores, tales como la variabilidad del ser observador, el momento de lectura, la calidad de las sustancias del test, la irradiación de la piel previa por luz ultravioleta, la administración de esteroides tópicos u orales y la fase del ciclo menstrual ${ }^{21}$.

Además de su indicación en la determinación de la DC alérgica, existen otras indicaciones para realizar pruebas epicutáneas como el eccema crónico y/o recidivante de manos resistente a los tratamientos indicados, el eccema atópico, que puede agravarse por una sensibilización a determinados alérgenos que se manipulan en el trabajo, la 
dermatitis seborreica, el eczema alrededor de ulceras de extremidades inferiores, o el exantema inducido por medicamentos ${ }^{12,21}$.

Las contraindicaciones para la realización de pruebas de contacto son: pacientes inmunodeprimidos o con dermatitis aguda, personas que reciban tratamiento inmunosupresor o inmunomodulador con glucocorticoides o ciclosporina, enfermedades autoinmunes, el embarazo y la lactancia ${ }^{12,22}$.

Las series estándar de pruebas epicutáneas son sustancias que se recopilan basadas en el área geográfica de exposición a haptenos determinados, características epidemiológicas, riesgo de exposición y sensibilización, y su correcta elección dependerá de las circunstancias de cada paciente. Es importante considerar que se van introduciendo nuevas substancias químicas en el entorno laboral y no laboral, por lo que estas series necesitan constante revisión y actualización. Una alta sospecha del origen ocupacional de una dermatosis se debe plantear cuando existen múltiples trabajadores afectados por dermatitis. Cuando se detecta una dermatosis, en el proceso de investigación es importante obtener información sobre la posible causa a través de las fichas de seguridad de los productos químicos que aporten los trabajadores ${ }^{23}$. Asimismo, es esencial para el médico entender como el trabajador se desempeña en su sitio de trabajo, como realiza sus tareas habituales, por lo que la visita al puesto de trabajo puede ser de gran utilidad ${ }^{5}$. Los problemas más comunes a los que se deben enfrentar los médicos es la distinción entre los cuadros de DC irritativa y alérgica, así como la relevancia de una reacción positiva en las pruebas epicutáneas.

La validez del test, así como su aportación diagnóstica dependerá de factores como: una historia clínica adecuada, una correcta aplicación de la batería de alergenos, el lugar de colocación (que debe ser una región amplia y desprovista de vello; como la espalda, cara interna del brazo, cara anterior del muslo y abdomen) ${ }^{22}$. En caso de existir vello se debe rasurar la zona a testar al menos 48 horas previas para evitar irritación ${ }^{24}$. Los efectos adversos pueden ser: a) irritación de la espalda cubierta por el esparadrapo, b) una respuesta exagerada a alguna sustancia alergénica, c) empeoramiento de la dermatitis, d) trastornos de la pigmentación, e) urticaria o anafilaxia y f) la potencial sensibilización a algún componente evaluado ${ }^{5}$. Basándose en los principios de medicina basada en la evidencia, la prueba de parche es rentable solo si los pacientes son seleccionados sobre la base de una sospecha clínica clara de alergia de contacto y a los pacientes se les prueban con productos químicos relevantes para el problema ${ }^{25}$. Las sustancias de composición desconocida, así como los productos que potencialmente son irritantes o tóxicos no deben ser evaluados tal cual, debiéndose hacer diluciones de los mismos $(10 \%, 5 \%$ y $1 \%)$ dependiendo de su toxicidad o $\mathrm{pH}$, ya que pueden generar una dermatitis irritativa necrótica e incluso una posible sensibilización activa ${ }^{5}$ (fig. 1). 
Figura 1. Localización de las Pruebas

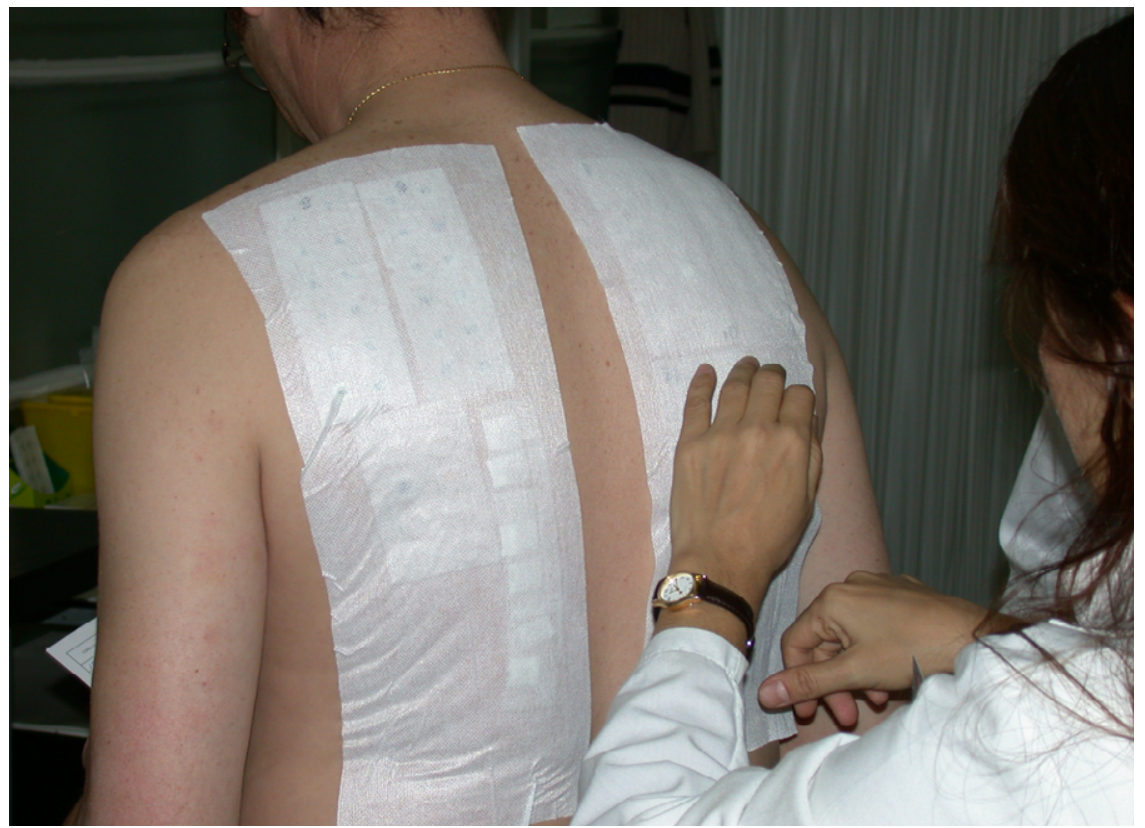

\section{RESULTADOS}

Para una adecuada interpretación se deben retirar los parches a las 48 horas y esperar al menos 30 minutos antes de realizar la primera lectura. Además se debe dejar marcado la zona de la piel donde estuvieron colocados los parches para las posteriores lecturas, que serán realizadas a las 72-96 horas y en algunas ocasiones hasta 5-7 días, dependiendo del alérgeno aplicado. En caso de encontrar una respuesta de urticaria se debe esperar mínimo de 60 minutos hasta 24 horas para realizar la primera lectura. La morfología de una prueba positiva presenta eritema, edema y pequeñas vesículas que se extienden ligeramente más allá de los bordes de la zona demarcada en la superficie del piel que ha estado en contacto con el alérgeno. La resolución rápida de la reacción a la prueba después de la retirada del parche es sugestiva de irritación ${ }^{26}$ (Tabla III).

Tabla III. Interpretación de los resultados de las pruebas de parche

\begin{tabular}{cllc}
\hline \multirow{2}{*}{ INFORME } & \multicolumn{1}{c}{ LECTURA } & \multicolumn{1}{c}{ INTERPRETACIÓN } & PRECISIón \\
\hline$+?$ & Eritema débil & Dudoso & $1 \%$ \\
+ & Eritema, infiltración y posiblemente pápulas & Posible & $20-50 \%$ \\
++ & Eritema, infiltración, pápulas y vesículas & Probable & $80-90 \%$ \\
+++ & Eritema intenso, infiltración y vesículas & Muy probable & $95-100 \%$ \\
& coalescentes & & \\
- & Ausencia de reacción & Negativo & \\
RI & & Reacción irritante & \\
NP & & No probada & \\
\hline
\end{tabular}

En general las reacciones irritativas ocurren de forma temprana y desaparecen rápidamente, mientras que las reacciones alérgicas exhiben un patrón creciente durante varios días. Es por este motivo que se recomienda hacer una lectura no solo al día dos, sino posteriormente ya que muchas reacciones irritativas pueden erróneamente determinarse como positivas ${ }^{1}$. 
En muchas ocasiones podemos encontrar una o más pruebas positivas. En estos casos se puede tratar que el enfermo se encuentre sensibilizado a diversos alérgenos, que bien se encuentran en un único producto que maneja el paciente, o que se encuentren en diversos productos utilizados en su vida profesional o privada. Otras veces puede darse que la aparición de diversas pruebas positivas sean debido a una reacción cruzada entre sustancias de composición química muy similar ${ }^{1,12,24}$ (figuras 2 y 3).

Figura 2. Demarcación de las pruebas

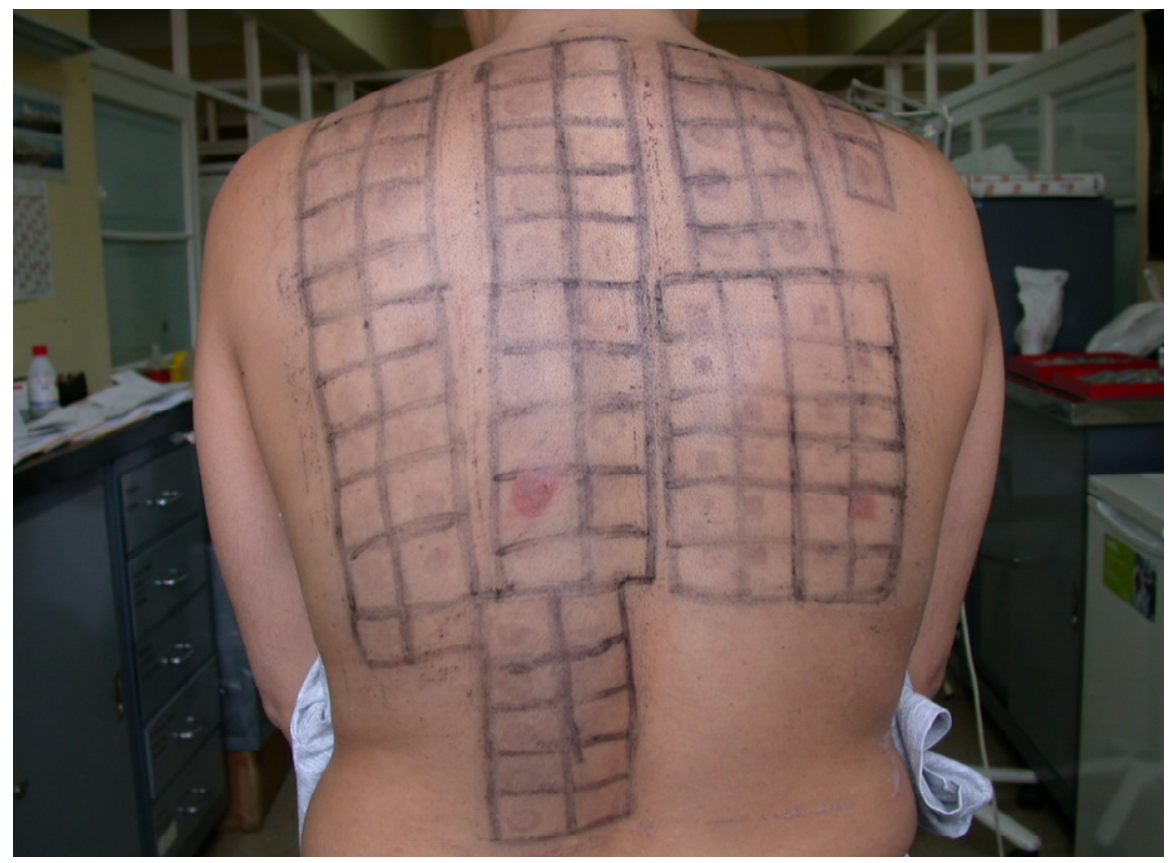

Figura 3. Prueba Positiva a para-fenilendiamina

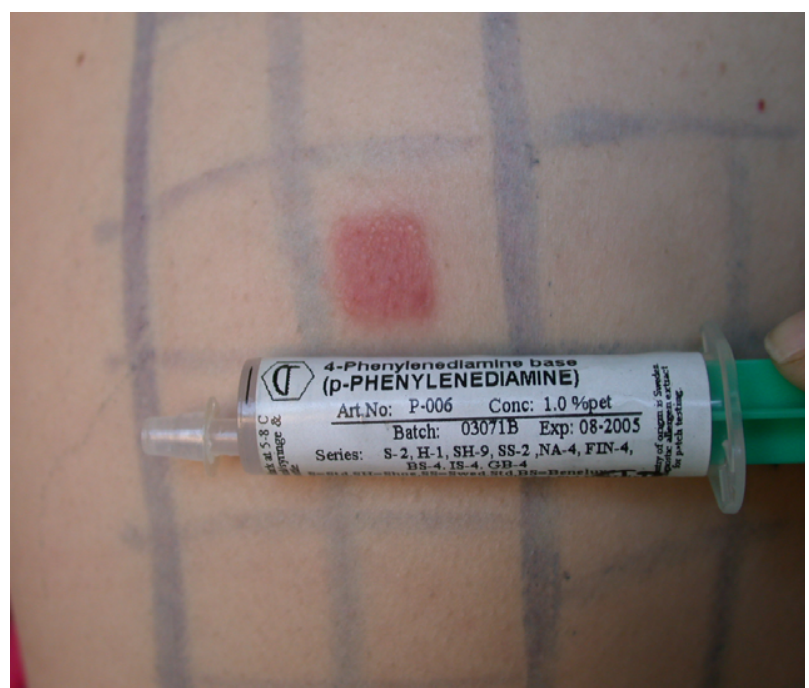

Finalmente, ante una prueba positiva es necesario e imprescindible buscar la relevancia de esta sensibilización con la historia clínica del enfermo. Esta puede ser actual cuando está relacionada con la historia actual del enfermo y es la causa de sus lesiones; relevancia pasada es, cuando la positividad no guarda relación con el cuadro cutáneo actual que presenta el enfermo, pero en la historia clínica se puede encontrar la posibilidad de antecedente de dicha sensibilización $\mathrm{y}$, relevancia desconocida se refiere cuando una positividad no tiene relación con el cuadro de lesiones actuales ni con ningún dato que aporte el enfermo en su historia. 


\section{CONCLUSIONES}

Las pruebas de contacto son muy útiles en Medicina Laboral y fundamentales en el diagnostico de las DC alérgicas. Su utilización servirá no solo para el diagnóstico, sino también para conocer la causa de la DC alérgica y con ello la posibilidad de tratar y de ubicar al enfermo o trabajador en otros puestos de trabajo donde no exista el contacto con dicho alérgeno y por tanto intentar evitar una incapacidad. Asimismo nos permite indicar al enfermo las substancias o productos de uso habitual no profesional que pueden ser causa de empeoramiento o de desencadenante de nuevas lesiones.

Además, las pruebas de contacto que son fáciles de realizar y de precio no muy costoso en comparación con otras técnicas diagnósticas. Pueden ser difíciles de interpretar en ocasiones, requiriendo personal perfectamente preparado para su realización, ya que de lo contrario una mala interpretación puede ser origen de problemas económicos y sociales importantes para el trabajador, la empresa y la sociedad en general.

Finalmente es importante resaltar que el Especialista en Medicina del Trabajo deberá conocer este tipo de pruebas dermatológicas y el valor que tienen a la hora de realizar un diagnóstico de dermatosis profesional, sin olvidar que constituye una herramienta más en el manejo de estos pacientes, que se interpreta tras un análisis profundo y conjunto de la historia clínica y profesional del paciente.

\section{REFERENCIAS BIBLIOGRÁFICAS}

1. Sasseville D. Occupational contact dermatitis. Allergy, Asthma and Clinical Immunology. 2008;4(2):59-65.

2. Rycroft, RJG. Frosch P. Occupational contact dermatitis. En: Frosch P, Menné T, Lepoittevin JP, editors. Textbook of contact dermatitis. London: Springer; 2006. 717.

3. Pere J, editor. Manual de Alergia Cutánea. 1st ed. España. MRA; 2011. p. 105-123.

4. Barchino Ortiz L, Cabrera Fernández E, Moreno Manzano G, Heras Mendaza F, Conde-Salazar Gómez L. Dermatosis profesionales en cuidadores de ancianos. Medicina y Seguridad del Trabajo 2007;53(207):35-46.

5. Gawkrodger DJ. Patch testing in occupational dermatology. Occup Environ Med. 2001;58(12):823-8.

6. De la Fuente I, Bell B, Ibarz J. Estudio epidemiológico de las dermatosis profesionales en el hospital MAZ (1993-1997). Revista Medicina del Trabajo. 1998;7(6):335,335-340.

7. Conde-Salazar L. Dermatologia Laboral En: Gil F, editor. Tratado de Medicina del Trabajo. Barcelona: Masson; 2005; p. 695.

8. Warshaw EM, Raju SI, Fowler JF, Maibach HI, Belsito DV, Zug KA, Rietschel RL, Taylor JS, Mathias C, Fransway AF. Positive patch test reactions in older individuals: Retrospective analysis from the north american contact dermatitis group, 1994-2008. J Am Acad Dermatol. 2012;66(2):229-40.

9. Lachapelle JM. Historical aspects. En: Duus J, Frosch P, Lepoittevin JP, editors. Textbook of contact dermatitis. London: Springer; 2011. p. 1-9.

10. Tato R, Sanz J, del Campo M, Agulló A. Dermatitis de contacto en el medio laboral. Medicina del Trabajo 1999;8(1):11-17.

11. Diepgen T, Kanerva L. Occupational skin diseases. Eur J Dermatol. 2006;16(3):324-30.

12. Conde-Salazar L, Ancona-Alayón A. Dermatología profesional. $1^{\mathrm{a}}$ Ed. Madrid: Grupo Aula Médica; 2004:51-63

13. Lachapelle J, Maibach HI. Patch testing methodology. En: Lachapelle J, Maibach HI, editors. Patch Testing and Prick Testing: A Practical Guide Official Publication of the ICDRG. London: Springer; 2012. p. 35-77.

14. Krasteva M, Kehren J, Ducluzeau MT, Sayag M, Cacciapuoti M, Akiba H, Descotes J, Nicolas JF, Moskophidis D, Lehmann-Grube F. Contact dermatitis. I. Pathophysiology of contact sensitivity. EJD. 1999;9:65-76.

15. Chew AL, Maibach HI. Occupational issues of irritant contact dermatitis. Int Arch Occup Environ Health. 2003;76(5):339-46

16. Dickel H, Altmeyer P, Brasch J. "New” techniques for more sensitive patch testing? JDDG: Journal der Deutschen Dermatologischen Gesellschaft. 2011;9(11):889-96. 
17. Dickel H, Kuss O, Schmidt A, Diepgen T. Occupational relevance of positive standard patch-test results in employed persons with an initial report of an occupational skin disease. Int Arch Occup Environ Health. 2002;75(6):423-34.

18. Van Der Valk PGM, Devos SA, Coenraads PJ. Evidence-based diagnosis in patch testing. Contact Derm. 2003;48(3):121-5.

19. Rietschel RL, Mathias C, Fowler Jr JF, Pratt M, Taylor JS, Sherertz EF, Marks Jr JG, Belsito DV, Storrs FJ, Maibach HI. Relationship of occupation to contact dermatitis: Evaluation in patients tested from 1998 to 2000. American Journal of Contact Dermatitis. 2002;13(4):170-6.

20. Bruze M, Conde-Salazar L, Goossens A, Kanerva L, White IR. Thoughts on sensitizers in a standard patch test series. Contact Derm 2007;41(5):241-250.

21. Spiewak R. Patch testing for contact allergy and allergic contact dermatitis. Open Allergy J. 2008;1:42-51.

22. Schnuch A, Aberer W, Agathos M, Becker D, Brasch J, Elsner P, Frosch PJ, Fuchs T, Geier J, Hillen U. Patch testing with contact allergens. JDDG: Journal der Deutschen Dermatologischen Gesellschaft. 2008;6(9):770-5.

23. Stevenson C. Occupational skin disease. Postgrad Med J. 1989;65(764):374.

24. Ardusso L. Prueba del parche. Enfoques en Asma y Alergia [en línea]. Asociacion Argentina de Alergia e Inmunologia Clínica. 2002; 3(1), 7-9. (Citado 2012 noviembre). Aproximadamente 3 pantallas. Disponible en: http://www.alergia.org.ar/profesionales/emc/enfoques_vol3_nro1_2002/enfoq6_7_9_2002.pdf.

25. Saint-Mezard P, Rosieres A, Krasteva M, Berard F, Dubois B, Kaiserlian D, Nicolas JF. Allergic contact dermatitis. EJD. 2004;14(5):284-95.

26. Krasteva M, Kehren J, Ducluzeau M, Sayag M, Dupuis M, Kanitakis J, Nicolas J. Contact dermatitis: II. clinical aspects and diagnosis. EJD. 1999;9:144-59. 